Trabajos y Comunicaciones, 2da. Época, No 48, e072, julio-diciembre 2018. ISSN 2346-8971

Universidad Nacional de La Plata.

Facultad de Humanidades y Ciencias de la Educación.

Departamento de Historia

\title{
Ofelia Rey Castelao y Pablo Cowen (editores) Familias en el Viejo y Nuevo Mundo Facultad de Humanidades y Ciencias de la Educación Universidad Nacional de La Plata, (HisMundi), 2017
}

\section{Elsa Caula *}

* Universidad Nacional de Rosario, Argentina elsacaula@hotmail.com

Cita sugerida: Caula, E. (2018). [Revisión del libro Familias en el Viejo y Nuevo Mundo por Ofelia Rey Castelao y Pablo Cowen (editores)]. Trabajos y Comunicaciones (48), e072. https://doi.org/10.24215/23468971e072 


\title{
Ofelia Rey Castelao y Pablo Cowen (editores) Familias en el Viejo y Nuevo Mundo
}

\author{
Elsa Caula \\ Universidad Nacional de Rosario, Argentina \\ elsacaula@hotmail.com
}

Entre muchas otras virtudes, este libro es producto de un trabajo colectivo dedicado a estudiar a las familias del Viejo y el Nuevo Mundo en el marco de las múltiples interacciones existente entre las dimensiones demográfica, económica, política, social y cultural. Esta perspectiva unificadora de los procesos que tienen como centro a las problemáticas familiares, permite redescubrir los variados espacios sociales en los que hombres y mujeres, grupos e instituciones desplegaron sus actividades y el significado que otorgaban a sus acciones, gracias a la utilización de metodologías renovadas y la utilización de un variado corpus documental entre los cuales la correspondencia epistolar tiene un protagonismo central. Este es precisamente uno de los aportes historiográficos más valiosos de este libro, resultado de las investigaciones realizadas en el marco de la Red Inter universitaria de Historia del Mundo Ibérico del Antiguo Régimen a las Independencias, que reúne a un numeroso grupo de destacados investigadores de las universidades nacionales argentinas de La Plata, Rosario y Mar del Plata y de las universidades españolas de Cantabria y País Vasco dedicados a los estudios de los procesos históricos desde la perspectiva de la familia. Quince artículos, cuyos autores y autoras son veinte especialistas en sus respectivos temas y forman parte activa en proyectos de investigación en sus respectivas universidades organizando seminarios donde discuten los resultados.

Ciertamente, con la finalidad de explicar y comprender las interconexiones de las familias del Viejo y del Nuevo Mundo y desde diferentes registros de análisis, los autores/as indagan en las prácticas y los discursos de actores diversos (los migrantes de las aldeas peninsulares hacia los centros nodales de la Monarquía y América, las familias judeocristianas, el alto clero, las familias campesinas, las mujeres de la elite, las monjas de clausura). Actores diversos, que son estudiados desde una perspectiva relacional y dialógica, prestando atención a los dispositivos institucionales y las herramientas jurídico-políticas que los distintos grupos sociales tuvieron a disposición para dialogar con las normas. Lo hacen desde diferentes reflexiones, ya sea desde la capacidad o no para resolver una conflictividad propia de los tiempos de cambios, poniendo en discusión los discursos científicos-jurídicos acerca del matrimonio y la moral social desde los cuales dicha conflictividad puede y conviene ser abordada; la cuestión de la venalidad, de la pureza de sangre, el asociacionismo como fórmula de establecimiento de redes de relaciones entre los emigrantes así como las diferentes expresiones y formas de vivir la familia.

El libro se inicia con el artículo de Francisco García González y Francisco Javier Crespo Sánchez titulado Radiografia de un impulso compartido. La historia de la familia en España e Iberoamérica (2000-2015) quienes realizan el recorrido historiográfico de los últimos años, subrayando los avances y los problemas del estado actual de la investigación. Durante estos tres lustros, los autores corroboran que uno de los aspectos que más ha sido estudiado el que vincula familia, negocio y poder, en especial, los aspectos referidos a las políticas familiares que proyectan sus expectativas sobre el Estado y la Iglesia como canales de movilidad social. Mientras que los temas referidos a la confrontación y el conflicto de las problemáticas familiares han sido menos elegidos y en consecuencia es donde más deben ser orientadas las nuevas investigaciones.

A continuación, el artículo de Rocío Sánchez Rubio e Isabel Testón Núñez se inscribe en las tendencias recientes de estudios de la escritura y su relación con la oralidad. Con el título El hilo que nos une. Las 
relaciones familiares y la correspondencia, las autoras consideran que los usos epistolares se propagaron y se divulgaron fundamentalmente como consecuencia de los grandes desplazamientos de población derivada de las numerosas guerras y, de manera especial, por la emigración transoceánica que llevó a los miembros de la familia a realizar aventuras ultramarinas. En el transcurso de los siglos XVI al XVIII -señalan- asistimos a una progresiva irrupción de la escritura en la cultura europea, favorecida por la popularización de los llamados egodocumentos, que se revelaron como el auténtico motor de cambio cultural en Europa. A lo largo del artículo se analizan varios corpus epistolares que pone en evidencia cómo la emigración a América jugó un papel fundamental en el desarrollo de las prácticas de la escritura en el ámbito de la monarquía hispánica, puesto que los individuos alejados de su tierra y sus seres queridos recurrían a las cartas como medio eficaz de mantener contacto a la distancia con sus familiares.

Seguidamente, Alberto Angulo Morales se ocupa de observar otros lazos también tangibles: los del asociacionismo como modo de establecer redes de relaciones entre los emigrantes norteños durante los siglos XVII y XVIII. Con el título Orden y desorden familiar en la emigración. El asociacionismo del norte peninsular (siglos XVII-XVIII), trazalos cambios producidos en el siglo XVIII en los procesos de migración de la aldea o la villa de origen a las principales ciudades de la Península y de América. El protagonismo de las antiguas cadenas migratorias dominadas por las familias notables dio lugar, según el autor, a la cooperación entre los diversos protagonistas involucrados en la movilidad poblacional a escala de monarquía creando un entramado relacional que permite comprender algunas de las claves del éxito de los hidalgos norteños tanto en América como en la Península.

A continuación, en la línea trazada por Jean Pierre Dedieu, Christían Windler y José María ImizcozBeunza hace algunos años, en relación a la importancia de las relaciones familiares en la constitución del cursushonorumde los agentes de gobierno de la monarquía hispánica, Francisco Andújar Castillo en Vínculos familiares entre el Viejo y el Nuevo Mundo: el aparato administrativo. 1674-1711,estudia el papel desempeñado por las familias para erigir a sus miembros al servicio de la administración y el gobierno de la monarquía hispánica en América.Destacando, la importancia de contextualizar el marco institucional que explique los cambios operados en dicha coyuntura, las formas de acceso a los cargos, el ejercicio del poder y el funcionamiento de los mecanismos político-administrativos por los que se proveía los cargos; precisamente porque el cambio de dinastía modificó pero no eliminó la venalidad de los cargos, que seguirá siendo una práctica política, nacida de la extensión de la vía ejecutiva o decisiva, esto es, la orden directa del rey a la Cámara de Indias, como modo derespetar su decisión de nombrar funcionarios sin consulta alguna. Por consiguiente, las vinculaciones familiares, de amistad, de paisanaje o patronazgo y entre "los dos mundos" no explicarían por si solas la provisión de cargos administrativos, puesto queel "mérito pecuniario" tuvo un protagonismo de primer orden entre el conjunto de recursos relacionales que articulaban la distribución de la gracia y la merced entre la Corte y los territorios americanos.

Con el título Elorigen judeoconverso de la nobleza indiana Enrique Soria Mesa describe en primer lugar las razones que explicarían el abandono historiográfico sobre el tema. Para resolver dicha desatención, estimula una vuelta a los archivos que guardan información abundante para trabajar en la restitución de la presencia de las familias judeoconversas entre las élites hispánicas silenciadas tanto por los historiadores americanos como por los españoles. Es importante agregar también que esta investigación, se inscribe en un proyecto dedicado a la nobleza y las elites de judeoconverso en Andalucía a partir del entrecruzamiento de fuentes documentales que permiten detectar las fallas en el control oficial sobre los pasajeros a Indias y la habilidad de quienes querían emigrar para eludir la normativa dictada por la monarquía.

A continuación, la dimensión femenina es abordada en el capítulo de María Luisa Candau Chacón titulado Madres e hijas. Familia y honor en la España moderna, en el que se analizan los textos de literatura didáctica y moral de los párrocos y moralistas, a partir de los discursos vigentes en la España católica. Con una metodología desde el enfoque de género la autora analiza tópicos que le permiten observar las identidades imaginadas en el seno de la familia, los roles atribuidos a las mujeres y la repartición de los espacios de estima 
social y legal que bajo el nombre de "honor" les correspondían a las mujeres en esa sociedad. Un orden moral y familiar característico de la España moderna que transmigró a ultramar de la mano de todos los grupos sociales que se establecieron en América.

Seguidamente, la importancia de las fundaciones conventuales como dispositivo de producción y reproducción de poder para el linaje y la familia, son estudiadas por Ángela Atienza López y José Luis Betrán Moya en Religiosos y religiosas. Lazos e intereses de familia en el seno del clero regular en el mundo hispánico de la Edad Moderna. La hipótesis que los guía sostiene que la fundación de conventos femeninos y masculinos por parte de los llamados indianos en España, fuente de patronazgo y de los lazos de unión con el Nuevo Mundo fue resultado de la divulgación y expansión del clero regular en correspondencia con el interés de las familias por consolidar el poder y ampliar sus redes de relaciones.

En el siguiente capítulo, Fernando Suárez Golán toma como objeto de estudio el alto clero en Familias de prelados: parientes, domésticos y comensales. En consonancia con los aportes de la historiografía francesa que sostiene que el sequito de los obispos jugó un rol decisivo en la afirmación episcopal frente a los otros poderes y dentro del estamento clerical, su investigación se ocupa de las familiaturas de los prelados, un colectivo que ha recibido poca atención en la historiografía española y americana. De ahí la importancia de sus valiosos aportes para conocer la peculiar conformación de la familia episcopal en torno a arzobispos y obispos, que se constituyeron en una verdadera red de influencia y cooptación, integrada tanto por lazos de sangre como de servidores domésticos y, sobre todo, por comensales y allegados que al amparo de sus patronos, se colocaban y prosperaban a ambos lados del Atlántico.

Pasando a los investigadores argentinos, Bibiana Andreuchi nos introduce en el tema de la desigualdad entre los distintos grupos sociales y etapas históricas, un tema central de la historia económica actual. Con el título Entre la ley y la práctica. Estrategias de transmisión del patrimonio en el Río de la Plata, siglos XVIII $y X I X$, la autora analiza en primer lugar, las estrategias usadas por las familias campesinas de la campaña bonaerense para conformar su patrimonio pecuniario, mantenerlo y/o aumentarlo; y, en segundo lugar, las destrezas adoptadas para transmitirlo a la siguiente generación, principalmente cuando entre los herederos había mujeres y niños, motivo por el cual fue habitual apartarse en la práctica de la norma sucesoria.

A continuación, en el marco del debate en torno al funcionamiento de la monarquía pluriterritorial y pluricéntrica española, que renovó el interés de la historia social por estudiar los comportamientos y la composición de las oligarquías urbanas, Carlos María Birocco se dedica a ahondar en el estudio del cabildo de Buenos Aires, una corporación concejil cuyos privilegios no se fundaban en la nobleza de sangre sino más bien en la riqueza. Con el título ¿Existieron en Buenos Aires linajes de cabildantes? Procedencia y parentesco en la dirigencia concejil porteña (1605-1726) muestra que la venalidad fue la modalidad de acceso a los oficios concejiles utilizada por la Corona como alternativa a la falta de nobleza de sangre. No obstantes destaca, que cuando se trataba de empleos electivos, los mecanismos de apropiación fueron más complejos ya que fue necesario conseguir el voto de los ascendientes de las familias para acceder al cargo.

A continuación, con el título Amor, matrimonio y procreación: sobre la teoría del amor en José Ingenieros, Cristina Beatriz Fernández analiza los modos de circulación de discursos y tópicos relacionados con los debates sobre las formas legalizadas de constitución familiar y la consolidación de una raza Argentina a partir del Tratado del amor, nacido como una serie de conferencias sobre la psicología de los sentimientos que José Ingenieros dictó en la Universidad de Buenos Aires en el año 1910 y luego adaptó para reeditarlos en forma de artículos en el marco de la serie La novela Semanal, una publicación que llegaba a públicos diversos ofreciendo "una visión secularizada y pretendidamente científica de las relaciones sentimentales y familiares, en una línea de reflexión sobre el amor, el matrimonio y la procreación tributaria de la moral anti dogmática, laica y modernizadora", que conciliaba las demandas de la eugenesia natural con el amor y al mismo tiempo desafiaba las convenciones sociales dominantes.

Seguidamente, el capítulo titulado Emociones, honra y familia. Comerciantes españoles a fines del siglo XVIII de Josefina Mallo estudia las manifestaciones de la honra familiar a finales del siglo XVIII desde una 
perspectiva metodológica novedosa. La autora, se introduce en la problemática del honor en el marco de las relaciones intrafamiliares para explorar las posibilidades de interpretación que brinda la historia de las emociones. El análisis está centrado en el intercambio de correspondencias entre los hermanos Callexas, dos establecidos en La Coruña y el tercero en Buenos Aires, sus hermanas y socios comerciales, nos introduce en el interior del ámbito doméstico, en la intimidad de las relaciones entre los miembros de la familia, donde la expresividad y el papel de las mujeres parece ser central.

A continuación, el capítulo de Osvaldo Otero Familias coloniales: vínculos, sentimientos y objetos Mundos intimos en una ciudad al borde del imperio española (1780-1820) examina las relaciones entre las formaciones familiares y la vivienda en la ciudad de Buenos Aires en el pasaje del orden colonial al independiente. Para el autor, las formas que adoptan la casa-familia, su estructura formal-funcional y sus objetos, son manifestaciones de una realidad compleja que tiene sus propias lógicas y merecen ser estudiadas. Así, al incluir en el estudio de la familia la vivienda familiar como objeto de estudio, permite conocer y comprender otras dimensiones de la intimidad familiar de los hombres y las mujeres que habitaron la ciudad de Buenos Aires.

Por último, el artículo titulado Alfaro Peñaloza-UrrejolaIzarza. Las familias de la transición al temprano orden borbónico en Santiago del Estero, 1700-1750 de María Cecilia Rossi nos lleva de Buenos Aires a Santiago del Estero, una de las ciudades que en esos años formaban parte del extenso Virreinato del Perú donde tras un intenso trabajo de relevamiento en archivos dispersos, comienza a reconstruir la/s historia/s familiar/es de tres generaciones de emigrantes de origen vasco que se fueron estableciendo en Santiago del Estero, una de las ciudades nodales en la ruta mercantil hacia el cerro rico de Potosí que estuvo conectada con el Atlántico mucho antes de la apertura del puerto de Buenos Aires al comercio directo con la metrópoli. En este capítulo se analiza en particular la rama familiar de los Alfaro y Peñaloza que los condujo a Urrejola Arzaza, el proceso de conformación del patrimonio familiar, las relaciones comerciales y la participación en las instituciones representativas de la monarquía en concordancia con los tempranos postulados borbónicos.

Para finalizar, me interesa destacar especialmente que el mérito adicional de esta obra es haber logrado el desafío que se propusieron como grupo de investigación en Red: ofrecer un encuadre óptimo para compendiar una publicación que nos acerca a los múltiples actores históricos que tienen como centro a las problemáticas familiares desde un enfoque compartido entre las experiencias históricas de los mundos ibéricos, que ha sido realizada en el marco de proyectos de investigación en las universidades nacionales argentinas y españolas bajo la coordinación de destacados y reconocidos especialistas. 